\title{
Property Evaluation of Bletilla striata/Polyvinyl Alcohol Nano Fibers and Composite Dressings
}

\author{
Jia-Horng Lin, ${ }^{1,2}$ Chao-Tsang Lu, ${ }^{3}$ Jin-Jia Hu, ${ }^{4}$ \\ Yueh-Sheng Chen, ${ }^{5}$ Chen-Hung Huang, ${ }^{6}$ and Ching-Wen Lou ${ }^{7}$ \\ ${ }^{1}$ Laboratory of Fiber Application and Manufacturing, Department of Fiber and Composite Materials, Feng Chia University, \\ Taichung 40724, Taiwan \\ ${ }^{2}$ School of Chinese Medicine, China Medical University, Taichung 40402, Taiwan \\ ${ }^{3}$ Graduate Institute of Biotechnology, Central Taiwan University of Science and Technology, Taichung 40601, Taiwan \\ ${ }^{4}$ Department of Biomedical Engineering, National Cheng Kung University, Tainan 70101, Taiwan \\ ${ }^{5}$ Department of Biomedical Imaging and Radiological Science, China Medical University, Taichung 40402, Taiwan \\ ${ }^{6}$ Department of Aerospace and Systems Engineering, Feng Chia University, Taichung 40724, Taiwan \\ ${ }^{7}$ Institute of Biomedical Engineering and Material Science, Central Taiwan University of Science and Technology, \\ Taichung 40601, Taiwan
}

Correspondence should be addressed to Ching-Wen Lou, cwlou@ctust.edu.tw

Received 19 June 2012; Accepted 25 September 2012

Academic Editor: Xiao-Miao Feng

Copyright ( 2012 Jia-Horng Lin et al. This is an open access article distributed under the Creative Commons Attribution License, which permits unrestricted use, distribution, and reproduction in any medium, provided the original work is properly cited.

\begin{abstract}
This study used nonwoven manufacture and electrospinning to create wound dressings with solid mechanical properties and hemostasis function. 10\% Polyvinyl alcohol (PVA) and 5\% Bletilla striata (BS) were blended into the PVA/BS solution, which can be made into nanomaterial with high specific surface area by electrospinning. The PVA/BS solution was electrospun onto the dressing matrix made of polyester (PET) and absorbent cotton (AC), forming the PVA/BS composite dressings. According to the experiment results, when the volume ratio of PVA to BS was $9: 1$, the resulting dressings had optimal fiber formation, the finest average diameter, and the lowest toxicity.
\end{abstract}

\section{Introduction}

Wound dressings are divided into gauze dressing, synthetic dressing, and biological dressing. Gauze dressings are composed of cellulose fibers or fur, such as gauze, cotton pad, or wool. This type of dressings is used to cover the wounds for a temporal protection and needs to be changed periodically. As various polymer materials have been developed as a result of chemical industry advances, there are many products for wounds; however, they are only good for skin-layer wounds, not for deep and large wounds [1-3].

In addition, sponge-form and spray-form synthetic dressings are available in the market; the design of synthetic dressings is further improved to be double-layer and multilayer, the former of which is composed of durable outer layer and adhesive and resilient inner layer. As all the aforementioned dressings do not significantly help large and deep burns, biological dressings with better quality and functionality are thus developed. An ideal dressing has to be able to keep wounds moist, to prevent the wounds from the second injury, to absorb the oozed blood and tissue fluid, to accelerate wound healing, as well as to have good resilience, tear strength, abrasion, and biocompatibility [4-8].

Electrospinning was first developed in 1900, when people placed melted sealing wax in an electric field, and solid fibers were jetted from the high-voltage end and accumulated on collector, forming membranes. Zeleny [9] explored the behavior of fluid droplets from a metal capillary under static electricity, and Formals (1934) published a complete electrospinning manufacturing that can successfully produce polymer filaments and that obtained the patent of electrospinning in US [10-12]. Fibers made by electrospinning are much finer than those by conventional spinning, yielding a diameter varying from tens to thousands of nanometers. 
The most desired functionality of electrospinning is to have nano fibers with considerable specific surface area, which could be largely applied in biomedicine, such as wound dressing, and tissue engineering scaffolding, tissue engineering vascular graft [13-16].

Polyvinyl alcohol (PVA), made from the hydrolyzed polyvinyl acetate, is primarily composed of hydrophobic acetyl group $\left(-\mathrm{CH}_{3} \mathrm{COO}\right)$ and hydrophilic hydroxyl group $(-\mathrm{OH})$. The side chain of molecular chain of PVA has a great amount of hydroxyl groups, imparting PVA great hydrophile. In addition, PVA also has good biocompatibility, nontoxicity, formability, and chemical and heat stability $[17,18]$. Bletilla striata (BS), a traditional Chinese medicine, is good for wound healing, hemostasis, detumescence, and granulation. It also helps to recover cuts, abrasion, toxin of wound tumescence, pulmonary hemorrhage, and gastric ulcer. Current studies have found that BS has glysan, volatile oil, and starch, which activate platelets, shorten time for thrombin generation, and suppress activity protease. Therefore, it shortens thrombin time, restricts fibrinolysis, and forms artificial thrombus so as to yield hemostasia [19-22].

Wounds, burns, surgical wounds, or acute wounds that have excessive bleeding demand an efficient wound dressings. In this study, PVA and BS solutions were first mixed with various volume ratios and then electrospun onto a PET/AC matrix, forming a hemostasic composite dressing. As PVA is able to complement BS for fiber formation, electrospinning is able to make polymer solution into micrometer fibers, and even nanometer fibers. The PVA/BS nano fibers differ from the previous PVA nano fibers in having the addition of Bletilla striata, which has functions of hemostasis and detumenscence. When electrospun into nano fibers, Bletilla striata has a high specific surface area; this increases the contact area between it and wounds, leading to an efficient treatment. The PVA/BS composite dressings thus had good mechanical properties, hemostasia, and biocompatibility. In addition, high specific surface area of the constituent PVA/BS nano fibers largely increased the contact area between BS and the wound, facilitating the hemostasia and healing. The PVA/BS nano fibers could be used as carrier for drug delivery, tissue engineering, tissue repair substitutes and wound dressing material.

\section{Experiment}

2.1. Material. PVA was purchased from Nihon Shiyaku Industries, Japan. BS was purchased from Fu Tan Pharmaceutical Co., Ltd., Taiwan. Deionized water was offered by Feng Chia University, Taiwan. L929 fibroblasts were supplied by Institute of Life Sciences, Taiwan.

2.2. Electrospinning. In the first stage, PVA powder and BS powder were respectively, dissolved with deionized water, formulating $10 \mathrm{wt} \%$ PVA solution and $5 \mathrm{wt} \%$ BS solution. Two solutions were then mixed with volume ratios of $10: 0$, $9: 1,8: 2,7: 3,6: 4,5: 5$, or $0: 10$ and then measured for viscosity. Afterward, these solutions were made into films and membrane by heating and electrospinning, respectively.

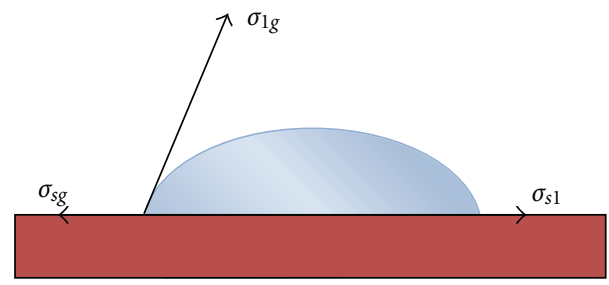

FIgURE 1: Illustration of contact angle.

The electrospinning machine was offered by the Laboratory of Fiber Application and Manufacturing, Feng Chia University, Taiwan. The settings of electrospinning were as follows: the voltage was $12 \mathrm{kV}$, the distance between the collector and needle was $12 \mathrm{~cm}$, the syringe flow was $0.75 \mathrm{~mL} / \mathrm{h}$. The films were evaluated in terms of contact angle while the membranes were observed by a scanning electron microscopy (SEM), according to which the optimal volume ratio of PVA to BS was thus determined.

In the second stage, the optimal volume ratio of PVA to BS was obtained from the first stage, the voltage of electrospinning was constant $(12 \mathrm{kV})$, the distance between collector and needle varied from $8,10,12,15$ to $20 \mathrm{~cm}$, and the syringe flow varied from $0.50,0.75$, to $1.00 \mathrm{~mL} / \mathrm{h}$. The resulting PVA/BS membranes were then observed by an SEM to determine the electrospinning parameters.

2.3. Preparation of Composite Dressings. The optimum parameters of fiber formation were used to in electrospinning process: PVA/BS mixture solution was electrospun on the optimal dressing matrix from the preliminary study [23], forming the PVA/BS composite wound dressings. The resulting samples were then evaluated in terms of tear strength, tensile strength, flexibility, and air permeability to determine the dressings' mechanical properties and comfort on users' skin.

\subsection{Tests}

2.4.1. Contact Angle Evaluation. Contact angle was based on Young's formula [24] proposed in 1805 as below:

$$
\sigma_{\mathrm{lg}} \operatorname{Cos} \theta=\sigma_{\mathrm{sg}}-\sigma_{\mathrm{sl}},
$$

where $\sigma_{\mathrm{lg}}$ was the interfacial tension between liquid and gas, $\sigma_{\mathrm{sg}}$ was the interfacial tension between solid and gas, $\sigma_{\mathrm{sl}}$ was the interfacial tension between solid and liquid, and $\theta$ was the contact angle, all of which were exemplified in Figure 1. PVA/BS solutions with various volume ratios were made into films and then evaluated by a contact angle meter (Kyowa Interface Science Co., Ltd., Japan). The number of test samples was six.

2.4.2. Observation of Scanning Electron Microscopy. PVA/BS solutions with various volume ratios were, respectively, electrospun into membranes, gilt, and then observed by a scanning electron microscopy (SEM, S3000N, Hitachi Ltd., Japan). 


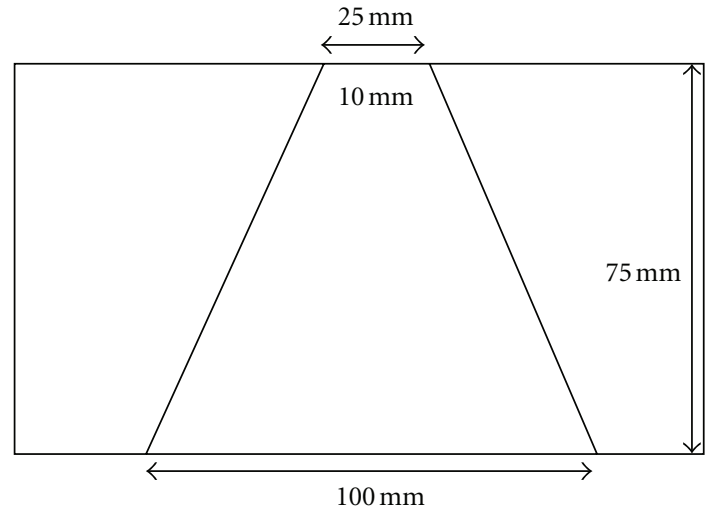

Figure 2: Sample of tear strength test.

2.4.3. Cell Biocompatibility Test. In in vitro test, PVA/BS membranes were tested for cell biocompatibility, as specified in ASTM F813-83. The membranes of $1 \mathrm{~cm} \times 1 \mathrm{~cm}$ were placed in a dish, where the L929 fibroblasts and medium were both infused. After cell culture, samples were observed for the cell growth.

2.4.4. Tensile Strength Test. This test was conducted according to CNS 12915, using a universal strength tester (HT9101, Hung Ta Instrument Co., Ltd., Taiwan). Ten composite dressings of $150 \mathrm{~mm} \times 25.4 \mathrm{~mm}$ were each taken along machine direction (MD) or cross machine direction (CD). The yields of values were averaged for definitive mean and standard deviation.

2.4.5. Tear Strength Test. A universal strength tester (HT9101, Hung Ta Instrument Co., Ltd., Taiwan, ROC) evaluated the tear strength of the composite dressings, as specified in CNS 12915. Figure 2 illustrates the sample size. Ten samples, each taken along the $\mathrm{CD}$ or $\mathrm{MD}$, were evaluated for the definitive mean and standard deviation.

2.4.6. Air Permeability Test. This test was performed as specified in ASTM D737, using an air permeability tester (FX 3300, Textest AG, Switzerland). The composite dressings were tested thirty times at different locations, and the values were then averaged for definitive mean.

2.4.7. Flexibility Test. A flexometer (Hung Ta Instrument Co., Ltd., ROC) was used to test the composite dressings, according to CNS 12915. Six samples of $150 \mathrm{~mm} \times 25.4 \mathrm{~mm}$, each taken along the MD and CD, were tested for definitive mean and standard deviation.

\section{Results and Discussion}

3.1. Contact Angle of Films Made by PVA/BS Solutions with Various Volume Ratios by Heating. Figure 3 shows the contact angles of PVA/BS films made with various volume ratios. With an increase in $\mathrm{BS}$ content, the contact angle of

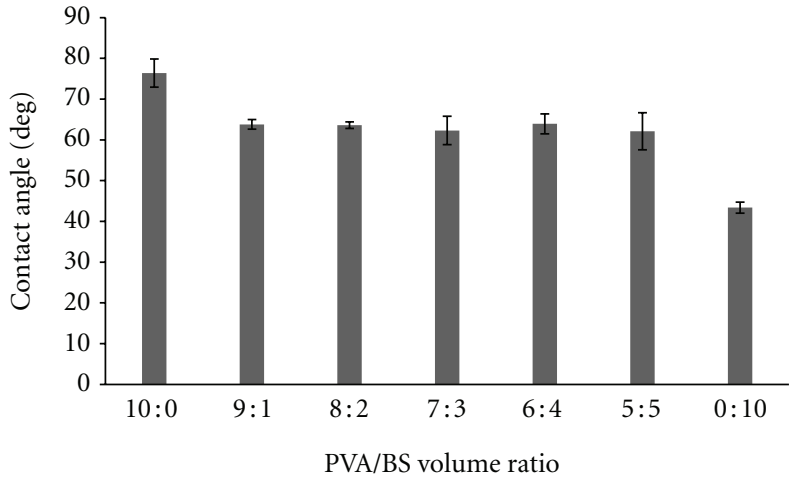

FIGURE 3: Contact angles as related to various PVA/BS volume ratios of the resulting films.

TABle 1: Mean of fiber diameter and viscosity of the PVA/BS solution as related to various BS contents.

\begin{tabular}{lcc}
\hline $\begin{array}{l}\text { BS content } \\
(\%)\end{array}$ & $\begin{array}{c}\text { Mean of fiber } \\
\text { diameter } \\
(\mathrm{Nm})\end{array}$ & $\begin{array}{c}\text { Viscosity of } \\
\text { PVA/BS solution } \\
(\mathrm{cp})\end{array}$ \\
\hline 0 & $181 \pm 41$ & 450.0 \\
10 & $179 \pm 28$ & 378.0 \\
20 & $177 \pm 35$ & 321.4 \\
30 & $164 \pm 41$ & 230.7 \\
40 & $212 \pm 34$ & 155.2 \\
50 & $256 \pm 38$ & 69.1 \\
100 & $\mathrm{~N} / \mathrm{A}$ & 41.7 \\
\hline
\end{tabular}

PVA/BS films decreased. This decrease was due to the fact that the molecular chains of PVA had a greater amount of chemicals found in the hydrophilic group $(-\mathrm{OH})$ and some found in the hydrophobic group $\left(-\mathrm{COOCH}_{3}\right)$; the major component of BS was carbohydrate, which was composed of carbon molecules and a great amount of certain hydrophilic chemicals $(-\mathrm{OH}$ and $-\mathrm{H})$. Therefore, an increase in BS content raised the amounts of hydrophilic groups in PVA/BS films, resulting in a greater hydrophile. This study shows that PVA/BS films were hydrophilic as the size of contact angle between the material and water was small.

3.2. SEM of Nanomembranes Made of PVA/BS Solutions with Various Volume Ratios by Electrospinning. Figure 4 shows the nano fibers made of PVA/BS solutions with volume ratios of $10: 1$ (pure PVA), 9:1, $8: 2,7: 3,6: 4$, and $5: 5$, by electrospinning. It was observed that when the BS content was increased, the electrospun fibers of PVA/BS solution exhibited distinct beads.

Table 1 reports the influence of various BS contents on the mean of fiber diameter and the viscosity of the PVA/BS solution. The mean of fiber diameter first decreased with an increase in the BS content, starting from 0, up to 10,20 , and $30 \%$; when BS content exceeded $30 \%$, the mean of fiber diameter then increased and was greater than that with $0 \%$ of BS content. An increase in BS 


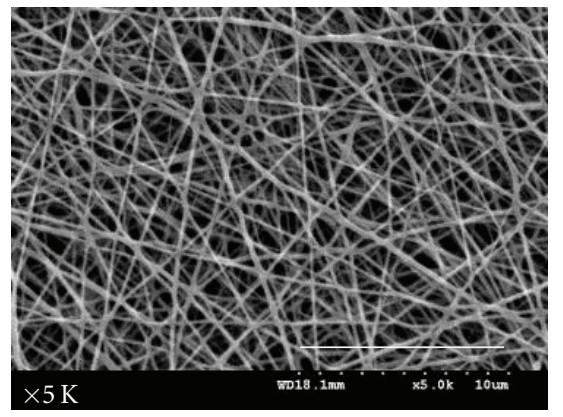

(a)

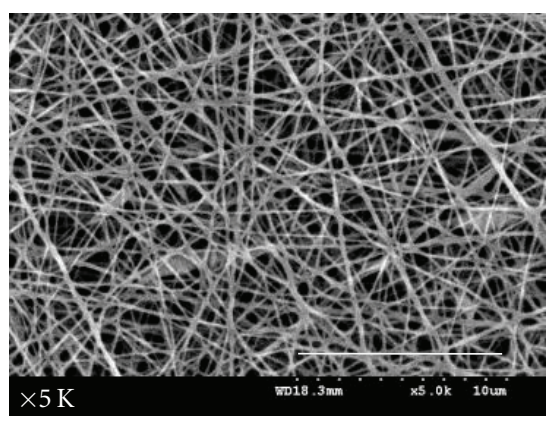

(d)

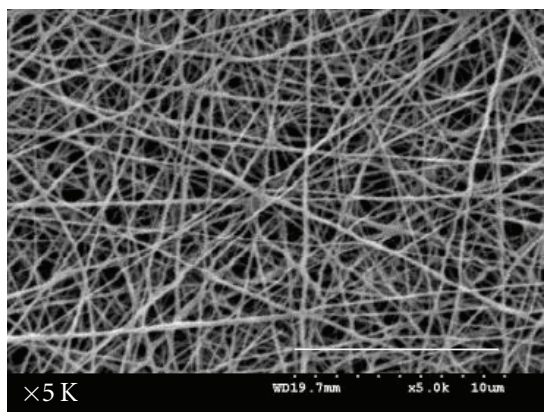

(b)

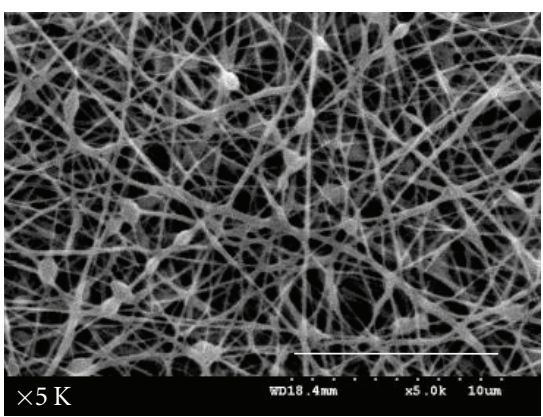

(e)

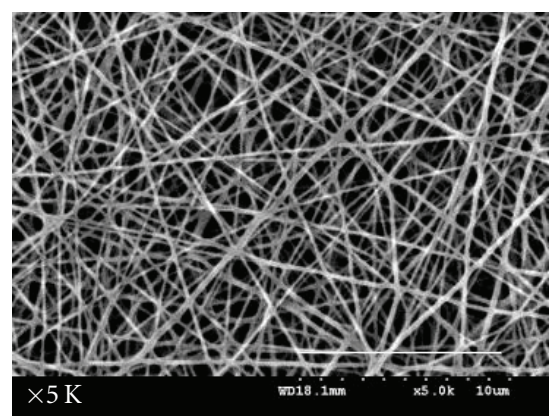

(c)

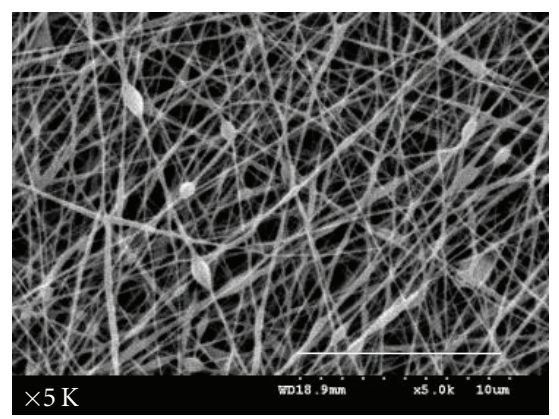

(f)

Figure 4: SEM images $(\times 5000)$ of nano fibers made of PVA/BS solution with volume ratios of (a) pure PVA, (b) $9: 1$, (c) $8: 2$, (d) $7: 3$, (e) $6: 4$, and (f) $5: 5$, by electrospinning. Scale bar: $10 \mu \mathrm{m}$.

content also decreased the viscosity of PVA/BS solution. Pure BS solution possessed a low viscosity, the greater the amount of BS content, the lower the viscosity of PVA/BS solution. The viscosity of PVA/BS solution was proportional to the mean of fiber diameter, namely, the mean of fiber diameter decreased with a decrease in the viscosity of PVA/BS solution.

According to Figure 4, beads started to occur when BS content was $20 \%$, indicating that the viscosity of PVA/BS solution was lower than the critical value of polymer viscosity required by electrospinning. In this study, the critical value of desired viscosity fell within $378.2 \sim 321.4 \mathrm{cp}$. The optimal volume ratio of $\mathrm{PVA} / \mathrm{BS}$ solution was $9: 1$, with which the resulting fibers exhibited no beads, resulting in the optimal fiber formation.

3.3. SEM of the PVA/BS Nano Fibers Made by Various Electric Fields and Syringe Flows. Table 2 reports the effect of various electric fields and syringe flows on the mean of fiber diameter of the electrospun fibers made of PVA/BS solution of $9: 1$, which was obtained from Section 3.2. The electric field varied from $0.6,0.8,1.0,1.2$ to $1.4 \mathrm{kV} / \mathrm{cm}$ while the syringe flow varied from $0.50,0.75$ to $1.00 \mathrm{~mL} / \mathrm{h}$. According to Table 2, five combinations of electric fields and syringe flows, which created a smaller mean of fiber diameter, were selected. Figure 5 shows the SEM images of the resulting PVA/BS fibers with the chosen five combinations
TABLE 2: Mean of fiber diameter as related to various electric fields and syringe flows.

\begin{tabular}{lcc}
\hline $\begin{array}{l}\text { Electric field } \\
(\mathrm{kV} / \mathrm{cm})\end{array}$ & $\begin{array}{c}\text { Syringe flow } \\
(\mathrm{mL} / \mathrm{h})\end{array}$ & $\begin{array}{c}\text { Mean of fiber diameter } \\
(\mathrm{Nm})\end{array}$ \\
\hline 0.6 & 0.50 & $206 \pm 46$ \\
0.8 & 0.50 & $170 \pm 29$ \\
1.0 & 0.50 & $222 \pm 34$ \\
1.2 & 0.50 & $247 \pm 39$ \\
1.4 & 0.50 & $186 \pm 35$ \\
0.6 & 0.75 & $195 \pm 30$ \\
0.8 & 0.75 & $249 \pm 43$ \\
1.0 & 0.75 & $179 \pm 28$ \\
1.2 & 0.75 & $239 \pm 40$ \\
1.4 & 0.75 & $220 \pm 36$ \\
0.6 & 1.00 & $193 \pm 37$ \\
0.8 & 1.00 & $192 \pm 41$ \\
1.0 & 1.00 & $227 \pm 38$ \\
1.2 & 1.00 & $243 \pm 36$ \\
1.4 & 1.00 & $199 \pm 45$ \\
\hline
\end{tabular}

of $\quad 0.6 \mathrm{kV} / \mathrm{cm}-1.0 \mathrm{~mL} / \mathrm{h}, \quad 0.8 \mathrm{kV} / \mathrm{cm}-0.5 \mathrm{~mL} / \mathrm{h}, \quad 0.8 \mathrm{kV} / \mathrm{cm}-$ $1.00 \mathrm{~mL} / \mathrm{h}, \quad 1 \mathrm{kV} / \mathrm{cm}-0.75 \mathrm{~mL} / \mathrm{h}$, and $1.4 \mathrm{kV} / \mathrm{cm}-0.5 \mathrm{~mL} / \mathrm{h}$. There was variation in the mean of fiber diameter because 


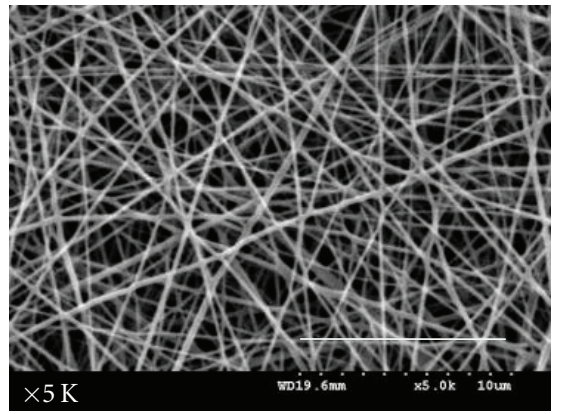

(a)

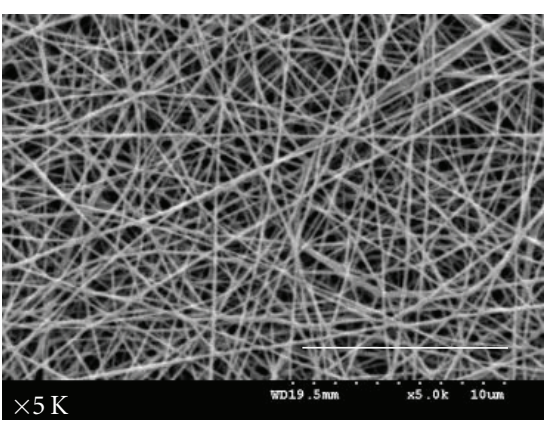

(b)

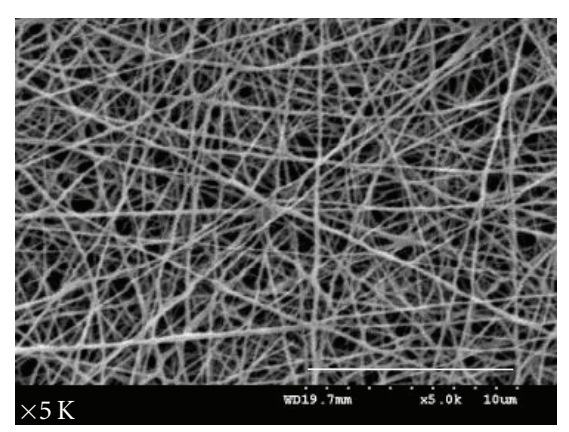

(c)

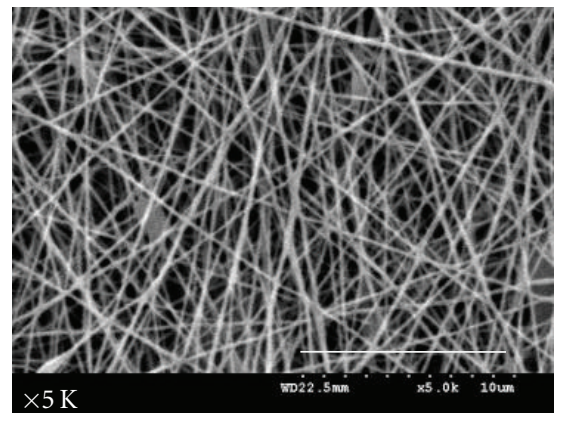

(d)

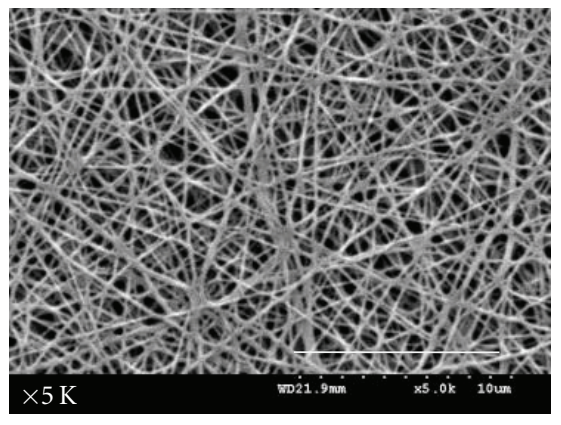

(e)

FIGURE 5: SEM images $(\times 5000)$ of electrospun fibers made of PVA/BS solution $(9: 1)$ with various combinations of electric field and syringe flow of (a) $0.6 \mathrm{kV} / \mathrm{cm}-1.0 \mathrm{~mL} / \mathrm{h}$, (b) $0.8 \mathrm{kV} / \mathrm{cm}-0.5 \mathrm{~mL} / \mathrm{h}$, (c) $0.8 \mathrm{kV} / \mathrm{cm}-1.00 \mathrm{~mL} / \mathrm{h}$, (d) $1 \mathrm{kV} / \mathrm{cm}-0.75 \mathrm{~mL} / \mathrm{h}$, and $(\mathrm{e}) 1.4 \mathrm{kV} / \mathrm{cm}-0.5 \mathrm{~mL} / \mathrm{h}$. Scale bar: $10 \mu \mathrm{m}$.

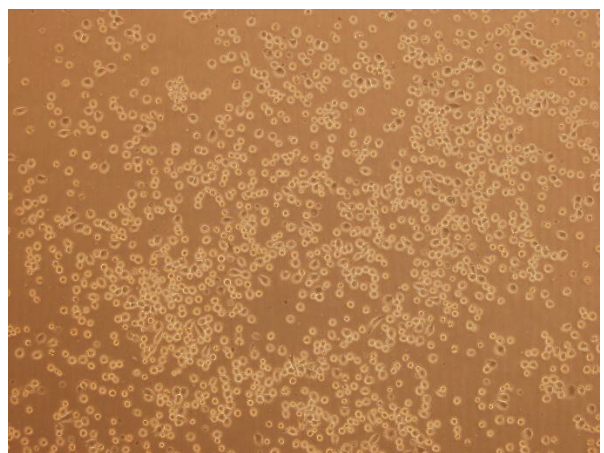

(a)

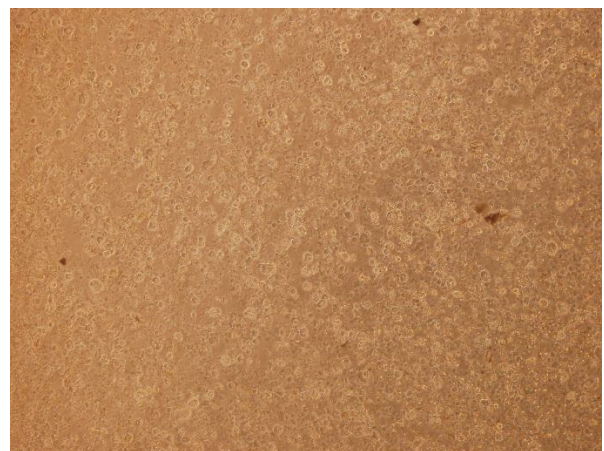

(b)

FIGURE 6: Optical microscope images of cell growth of (a) control group (pure L929 fibroblasts cultured in medium), and (b) L929 fibroblasts cultured with a PVA/BS nanomembrane in medium.

when the charge was varied, the polymer solution was turned into different shapes, called Taylor cones, on the tip of the syringe as a result of an imbalance of surface tension of polymer solution (repulsion force) and electric field force (pulling force). This influenced the generated drops, fiber diameter, fiber morphology, and the strength of currents.

With the five given combinations of electric fields and syringe flows, the resulting Taylor cones did not create beads, showing that these Taylors cones, formed during electrospinning, were all suitable for fiber formation. In addition, variation in electric fields generated currents with various strengths during currents' transmission in polymer solution, forming PVA/BS nano fibers with various diameters. The optimal combination was thus determined to be an electric field of $0.8 \mathrm{kV} / \mathrm{cm}$ and a syringe flow of $0.5 \mathrm{~mL} / \mathrm{h}$, which produced the smallest mean of fiber diameter $(170 \pm 29 \mathrm{~nm})$; the amount of fibers was also greater than the other groups.

3.4. Cell Biocompatibility Test. Figure 6 shows the images of cell growth magnified 40 times: (a) is the control group (pure L929 fibroblasts cultured in medium), and (b) is L929 fibroblasts cultured with a PVA/BS nanomembrane in medium. Comparing Figures 6(a) and 6(b), the cell amount did not 
TABLE 3: Comparison of mechanical properties of PVA/BS composite dressings and PET/AC matrices.

\begin{tabular}{lcccc}
\hline & \multicolumn{2}{c}{ PVA/BS } & \multicolumn{2}{c}{ PET/AC } \\
& composite dressings & \multicolumn{2}{c}{ matrices } \\
& CD & MD & CD & MD \\
\hline Maximum tear strength (N) & 106.7 & 79.8 & 103.6 & 76 \\
Standard deviation & 5.32 & 2.88 & 4.4 & 1.62 \\
Maximum tensile strength (N) & 130.7 & 38.1 & 123.9 & 36.8 \\
Standard deviation & 6.71 & 0.49 & 4.71 & 2.44 \\
Flexibility (cm) & 12.2 & 10.9 & 8.5 & 7.1 \\
Standard deviation & 0.65 & 0.45 & 0.16 & 0.33 \\
\hline
\end{tabular}

TABle 4: Comparison of air permeability of PVA/BS composite dressings and PET/AC matrices.

\begin{tabular}{lcc}
\hline & $\begin{array}{c}\text { PVA/BS composite } \\
\text { dressings }\end{array}$ & $\begin{array}{c}\text { PET/AC } \\
\text { matrices }\end{array}$ \\
\hline Air permeability $\left(\mathrm{cm}^{3} / \mathrm{s} / \mathrm{cm}^{2}\right)$ & 57.4 & 106.05 \\
Standard deviation & 9.39 & 4.55 \\
\hline
\end{tabular}

decrease largely when cells were cultured with a PVA/BS nanomembrane, indicating the resulting nanomembrane did not negatively interfere with cell growth. In other words, PVA/BS nanomembranes had a minor toxicity, resulting in only a minor cell loss after implantation of the nanomembranes.

\subsection{Mechanical Properties of PVA/BS Composite Dressings and} PET/AC Matrices. Tables 3 and 4 compare the tear strength, tensile strength, flexibility, and air permeability of PVA/BS composite dressings and PET/AC matrices. According to Table 3, after PVA/BS solution was electrospun to PET/AC matrices, the resulting composite dressings displayed greater mechanical properties. This was because electrospinning transformed the solution into a nanofibrous net, the nano fibers of which had a smaller diameter than that of PET/AC fibers. The nano fibers fell in and then filled the voids of PET/AC matrices. In addition, nano fibers also had a larger specific surface area, enlarging the contact area between the PVA/BS nano fibers and PET/AC fibers and, thus, raising the friction between fibers. An increase in friction contributed to a greater resistance for fibers to move. Therefore, to electrospin PVA/BS solution onto the PET/AC matrices indirectly imparted greater mechanical properties to the resulting PVA/BS composite dressings.

In contrast, the air permeability of the resulting PVA/BS composite dressings was lower than that of PET/AC matrices. Due to the aforementioned explanation about the distribution of nano fibers during electrospinning, the voids in PET/AC matrices were clogged by nano fibers, decreasing the air volume that passed through the voids per unit time, and the air permeability. However, the air permeability of PVA/BS composite dressings was still satisfactory.

\section{Conclusion}

This study proposed a PVA/BS composite dressing, which was made by electrospinning the PVA/BS solution onto the PET/AC matrices. The fiber formation and physical properties of the resulting materials as related to various parameters were explored. When PVA/BS solution had a volume ratio of $9: 1$, the critical value of the solution viscosity fell between 378.2 321.4 cp, creating no beads between the resulting PVA/BS nano fibers and thus providing the optimal fiber formation. With a constant volume ratio of $9: 1, \mathrm{PVA} / \mathrm{BS}$ solution could be electrospun into nano fibers with the smallest mean of fiber diameter $(170 \pm 29 \mathrm{~nm})$ when the electric field was $0.8 \mathrm{kV} / \mathrm{cm}$ and the syringe flow was $0.5 \mathrm{~mL} / \mathrm{h}$. In the cell biocompatibility test, PVA/BS nanomembranes had minor toxicity, which caused only insignificant cell loss. In comparison with PET/AC matrices, PVA/BS composite dressings were composed of nano fibers, which had a much smaller diameter and high specific surface area, and thus exhibited greater tensile and tear strength, flexibility, and a lower air permeability.

\section{Acknowledgment}

The authors are thankful to the National Science Council, Taiwan, ROC, for providing financial assistance to carry out this research work under NSC-97-2622-E-166-003-CC3.

\section{References}

[1] N. S. Levine, R. A. Lindberg, R. E. Salisbury, A. D. Mason Jr., and B. A. Pruitt Jr., "Comparison of coarse mesh gauze with biologic dressings on granulating wounds," American Journal of Surgery, vol. 131, no. 6, pp. 727-729, 1976.

[2] G. Linda and M. Phillips, "Wound healing," in Sabiston Textbook of Surgery, 16th edition.

[3] M. Malmsjö, R. Ingemansson, R. Martin, and E. Huddleston, "Negative-pressure wound therapy using gauze or open-cell polyurethane foam: similar early effects on pressure transduction and tissue contraction in an experimental porcine wound model," Wound Repair and Regeneration, vol. 17, no. 2, pp. 200-205, 2009.

[4] M. McGuckin, R. Goldman, L. Bolton, and R. Salcido, "The clinical relevance of microbiology in acute and chronic wounds," Advances in Skin \& Wound Care, vol. 16, no. 1, pp. 12-23, 2003.

[5] M. J. Morykwas, L. C. Argenta, E. I. Shelton-Brown, and W. McGuirt, "Vacuum-assisted closure: a new method for wound control and treatment: animal studies and basic foundation," Annals of Plastic Surgery, vol. 38, no. 6, pp. 553-562, 1997.

[6] M. J. Morykwas, L. C. Argenta, E. I. Shelton-Brown, and W. McGuirt, "Vacuum-assisted closure: a new method for wound control and treatment: animal studies and basic foundation," Annals of Plastic Surgery, vol. 38, no. 6, pp. 563-577, 1997.

[7] M. J. Morykwas and L. C. Argenta, "Nonsurgical modalities to enhance healing and care of soft tissue wounds," Journal of the Southern Orthopaedic Association, vol. 6, no. 4, pp. 279-288, 1997.

[8] T. A. Mustoe, "Evolution of silicone therapy and mechanism of action in scar management," Aesthetic Plastic Surgery, vol. 32, no. 1, pp. 82-92, 2008. 
[9] J. Zeleny, "The electrical discharge from liquid points and a hydrostatic method of measuring the electric intensity at their surface," Physical Review, vol. 3, no. 2, pp. 69-91, 1914.

[10] A. Formhals, "Process and Apparatus for Preparing Artificial Threads," US Patent, 1, 975, 504, 1934.

[11] A. Formhals, "Method and Apparatus for Spinning," US Patent, 2, 160, 962, 1939.

[12] A. Formhals, "Artificial Thread and Method of Producing Same," US Patent, 2,187,306, 1940.

[13] S. Y. Gu, J. Ren, and G. J. Vancso, "Process optimization and empirical modeling for electrospun polyacrylonitrile (PAN) nanofiber precursor of carbon nanofibers," European Polymer Journal, vol. 41, no. 11, pp. 2559-2568, 2005.

[14] K. T. Shalumon, N. S. Binulal, N. Selvamurugan et al., "Electrospinning of carboxymethyl chitin/poly(vinyl alcohol) nanofibrous scaffolds for tissue engineering applications," Carbohydrate Polymers, vol. 77, no. 4, pp. 863-869, 2009.

[15] Y. T. Jia, J. Gong, X. H. Gu, H. Y. Kim, J. Dong, and X. Y. Shen, "Fabrication and characterization of poly (vinyl alcohol)/chitosan blend nanofibers produced by electrospinning method," Carbohydrate Polymers, vol. 67, no. 3, pp. 403-409, 2007.

[16] N. Charernsriwilaiwat, P. Opanasopit, T. Rojanarata, T. Ngawhirunpat, and P. Supaphol, "Preparation and characterization of chitosan-hydroxybenzotriazole/polyvinyl alcohol blend nanofibers by the electrospinning technique," Carbohydrate Polymers, vol. 81, no. 3, pp. 675-680, 2010.

[17] S. E. Shih, Textile Physics, Taiwan Textile Research Institute, Taipei, Taiwan, 2000.

[18] C. A. Lin, "Fiber Chemistry," Taiwan Silk \& Filament Weaving Industrial Information Web, 2006.

[19] H. Diao, X. Li, J. Chen et al., "Bletilla striata polysaccharide stimulates inducible nitric oxide synthase and proinflammatory cytokine expression in macrophages," Journal of Bioscience and Bioengineering, vol. 105, no. 2, pp. 85-89, 2008.

[20] X. Miao, Shennong Ben Cao Jing Su, China Press of Traditional Chinese Medicine, Beijing, China, 1997.

[21] Shi-zhen Li, Pen Ts'ao Kang Mu, China Press of Traditional Chinese Medicine, Beijing, China, 1998.

[22] A. Wang, Pen Ts'ao Pei Yao, China Press of Traditional Chinese Medicine, Beijing, China, 1998.

[23] C. W. Lou, J. J. Hu, C. C. Huang, C. T. Lu, C. T. Hsieh, and J. H. Lin, "Preparation and characterization of polyester fibers/absorbent cotton composite dressing matrix fabrics," Advanced Materials Research, vol. 287-290, pp. 2721-2724, 2011.

[24] N. K. Adam, "Use of the term "Young's Equation" for contact angles," Nature, vol. 180, no. 4590, pp. 809-810, 1957. 

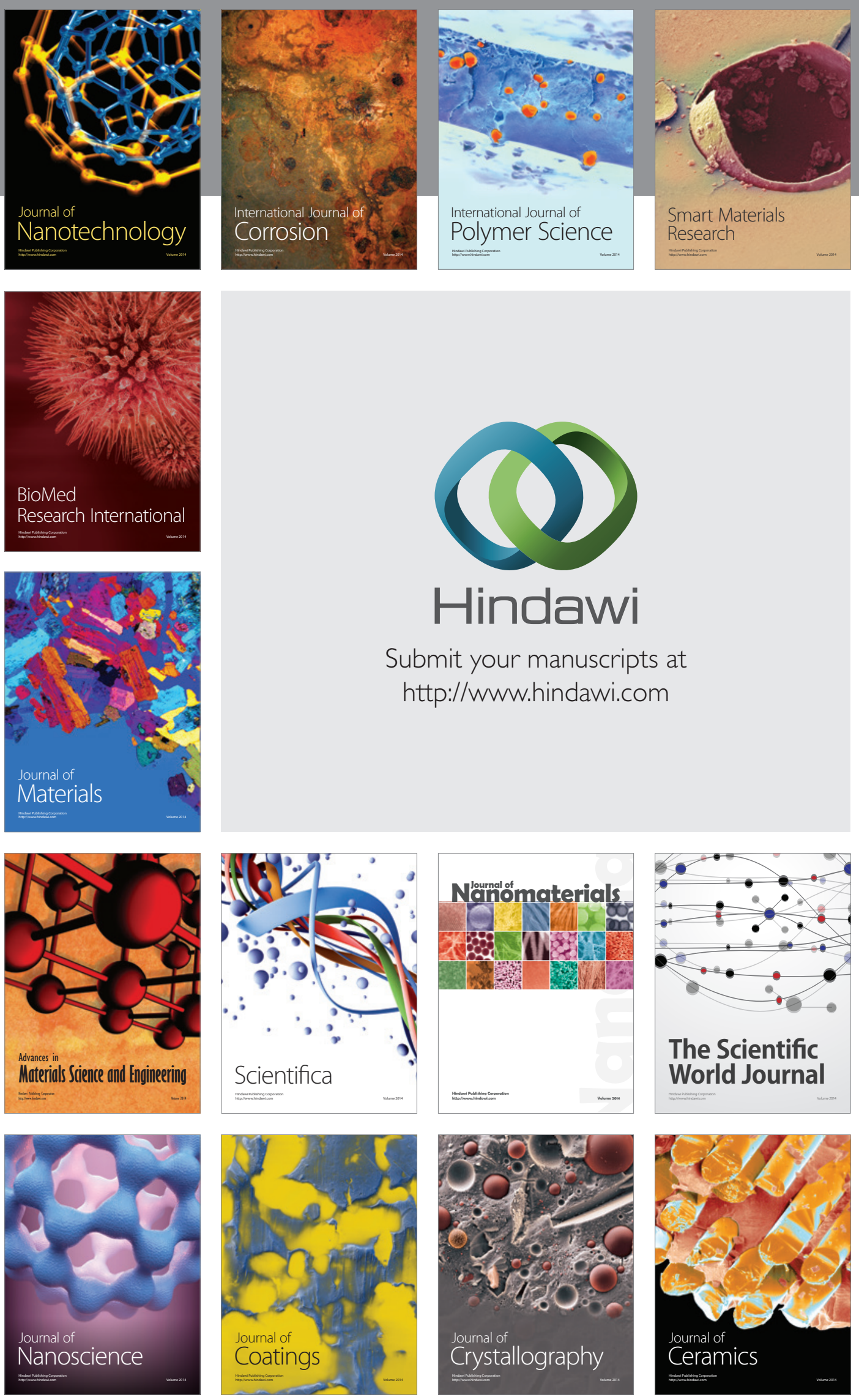

The Scientific World Journal

Submit your manuscripts at

http://www.hindawi.com

\section{World Journal}

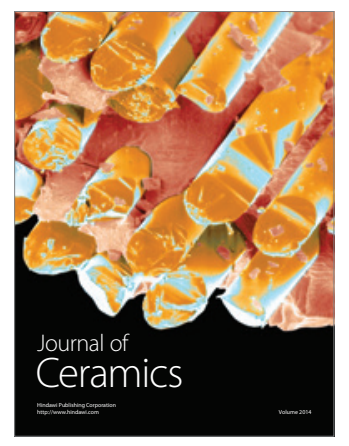

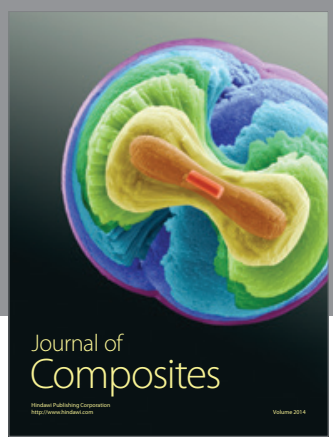
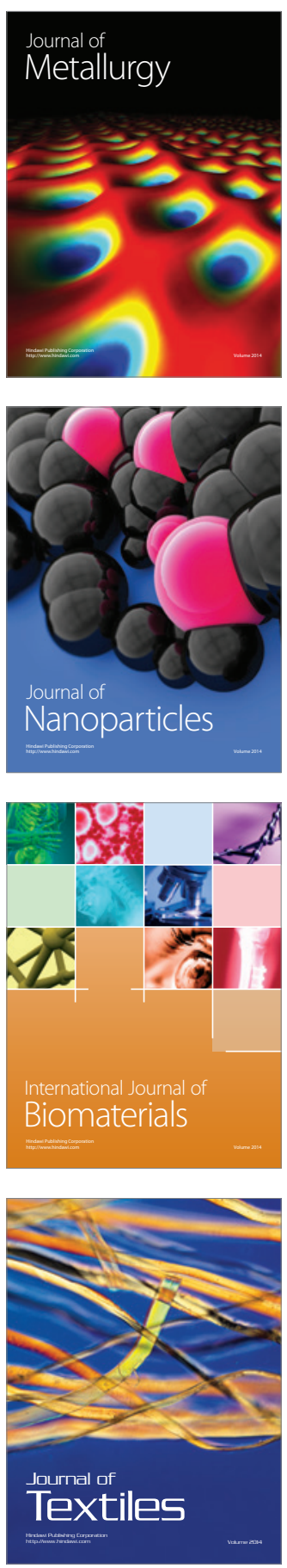